\title{
Summer schools for magnetic crystallography and neutron diffraction
}

\author{
E Rodriguez ${ }^{1}$ \\ ${ }^{1}$ University of Maryland, College Park, MD \\ efrain@umd.edu
}

Since it is not typically part of the syllabus for crystallagraphy courses, the topic of neutron diffraction can nicely be supplemented through summer schools. Even less discussed is a special application of neutron diffraction in the area of magnetism. I will discuss the summer schools that I have participated in aimed at graduate students and even post-doctoral research associates. The audience can be diverse including chemists, materials scientists and physicists, and this variety in background can pose a particular challenge for selecting the right level of background material. I will discuss the two different methods for teaching how to solve magnetic structures using neutron diffraction.

Acta Cryst. (2020). A76, a120 